\title{
Hypertension in Pregnancy and Related Health Outcomes in European Ethnic Minorities
}

\author{
Alessandra Modesti and Felice Petraglia
}

\subsection{Pregnancy Complications at the Global Level}

According to the data provided by the Global Health Observatory of the WHO, every day in 2015, about 830 women died due to complications of pregnancy and child birth. Almost all of these deaths occurred in low-resource settings and are related to pregnancy complications that are inadequately managed because of a lack of access to emergency health care. More precisely, of the 830 daily maternal deaths, 550 occurred in sub-Saharan Africa (SSA) and 180 in Southern Asia (SA), compared to 5 in wealthy nations [1]. In particular the maternal mortality ratios (MMRs) of Sweden, the United Kingdom, and the United States are 4, 12, and 21, respectively, whereas those of Chad, Nigeria, and Congo are 1100, 630, and 540 per 100,000 live births, respectively. The primary causes of death are haemorrhage, hypertension, infections, and indirect causes, mostly due to interaction between preexisting medical conditions and pregnancy [2]. These high maternal and associated neonatal mortality rates persist despite considerable efforts from the World Health Organization, governments, development partners, and others [3]. Furthermore it is now evident that disparities in pregnancy and child birth are also present among minority groups living in Europe. In wealthy countries, women from minority racial groups have a higher prevalence of a wide range of adverse pregnancy outcomes, including miscarriage [4], stillbirth [5], pre-eclampsia (PE) [6-8], gestational

\footnotetext{
A. Modesti ( $\square)$

Department of Clinical and Experimental Medicine, University of Florence, Firenze, Italy e-mail: alessandra.modesti@unifi.it

F. Petraglia

Division of Obstetrics and Gynecology, Department of Experimental and Clinical Biomedical Sciences "Mario Serio", University of Florence, Florence, Italy

e-mail: felice.petraglia@unifi.it
} 
hypertension (GH) [9], gestational diabetes mellitus (GDM) [9-11], preterm delivery (PTD) [9, 12], delivery of small-for-gestational-age (SGA) [13, 14] or large-forgestational-age (LGA) [15, 16] neonates, and elective or emergency Caesarean section (CS) [17]. Apart from the obvious, cultural, and socioeconomic factors and different priorities in health care, additional biological reasons might be responsible for pre-eclampsia syndromes being such a prominent feature of African obstetrics. Hypertension developing in the second half of pregnancy is subdivided according to the presence or absence of co-existing significant proteinuria into pre-eclampsia and gestational hypertension. These two aspects are therefore important for ethnic minorities living in wealthy countries.

\subsection{Pre-eclampsia in Ethnic Minorities}

\subsubsection{Pre-eclampsia}

Pre-eclampsia, defined by the International Society for the Study of Hypertension in Pregnancy as blood pressure $\geq 140 / 90 \mathrm{mmHg}$ and 24 -h proteinuria $\geq 0.3 \mathrm{~g}$, is a multisystem disorder characterized by abnormal vascular response to placentation [18]. The syndrome is characterized by proteinuria and hypertension in a previously normotensive woman after gestational week 20 and occurs with a 2-8\% incidence of all pregnancies [19]. Pre-eclampsia is a leading cause of maternal morbidity and mortality globally, accounting for an estimated 50,000 maternal deaths annually [19]. An important determinant of pre-eclampsia is failure of placentation, particularly the physiological transformation of spiral arteries, which leads to a stressed, underperfused placenta [20]. Pre-eclampsia is a recognized clinical entity characterized by new onset of hypertension and proteinuria after 20 weeks' gestation [19, 21, 22]. In SSA the distinction between true pre-eclampsia and pregnancy-induced hypertension is sometimes difficult because proteinuria may not be adequately measured. A further problem is a lack of information on pre-existing hypertension because presentation to the clinic is often late. Data on pre-eclampsia in African Americans (AA) and immigrants from Africa to other high-income countries as compared with other ethnic groups are now available.

In the United States, Black ethnicity is cited as a risk factor for pre-eclampsia [23]. Of four million births recorded in the National Vital Statistics Report, pregnancy-associated hypertension was more common in AA (5.0\%) and least frequent in Hispanics (2.9\%), and AA women had the highest risk for all the different types of pre-eclampsia when compared with European American women [24]. In a 10-year longitudinal population-based study aimed at investigating ethnic disparity in hypertensive disorders of pregnancy in New York State, AA women had OR 1.67 (1.64 to 1.71) for pre-eclampsia vs. White women [25]. Poor socioeconomic status with lower incomes and level of education, lack of medical insurance, poor utilization of preconception and antenatal services, stress, discrimination, and residential segregation were often reported as possible explanations. However, many of the socioeconomic factors that may contribute to poor obstetric outcomes also apply to 
the Hispanic population in the United States, yet several studies have noted that preeclampsia, low birth weight, and stillbirth are similar or even better than for White women, the Hispanic paradox [7, 26].

Obstetric outcomes for recent African immigrants in Europe are informative, particularly because these births often take place in countries with good records and universal health-care systems [8]. In the Netherlands, the highest risk for eclampsia and pre-eclampsia was from women from SSA [27, 28]. Cape Verdean and Antillean women were also at higher risk of pre-eclampsia in a report from Rotterdam, the Netherlands [29].

In a large retrospective study in women with singleton pregnancies attending their first routine hospital visit, 80,000 pregnancies that included women of European and Asian ancestry were considered [30]. In Afro-Caribbean women, compared with Caucasians, there was a higher prevalence of miscarriage, stillbirth, pre-eclampsia, gestational hypertension, small-for-gestational age, spontaneous and iatrogenic preterm delivery, and emergency Caesarean section [30]. South Asian racial origin was associated with increased risk for gestational diabetes mellitus, pre-eclampsia, small-for-gestational age, iatrogenic preterm delivery, and Caesarean section, and East Asian race had increased risk for gestational diabetes mellitus and small-for-gestational age [30]. Similarly, in a prospective screening study for hypertensive disorders in women attending for their routine first hospital visit in pregnancy performed in the United Kingdom, Black women were found at increased risk of early pre-eclampsia, both Black and South Asian women being at increased risk for late pre-eclampsia [31]. Predictors of early pre-eclampsia were Black race, chronic hypertension, prior PE, and use of ovulation drugs [31]. Late pre-eclampsia was more common in Black, Indian, and Pakistani women [31]. After a woman has had pre-eclampsia in her first pregnancy, the risk of recurrence is increased, with a relative risk of 15.0 in an authoritative Norwegian study of more than two million women [32]. Other reports support these findings [8].

\subsubsection{Genetics of Pre-eclampsia}

A genetic component to pre-eclampsia has long been suspected on the basis of a familial recurrence of the syndrome. Daughters of women with pre-eclampsia have more than twice the risk of developing the disease themselves, and sisters of affected women, even if not born from a pre-eclamptic pregnancy, are also at increased risk $[33,34]$. Although environmental factors, particularly influences acting in utero, are important, some of the risk is likely to be genetic. Using ancestry informative markers, a case-control study of pre-eclampsia in Latinas, a group with admixture from European, African, and native Americans, showed that African ancestry was associated with pre-eclampsia [35]. Many reports indicate also a paternal contribution to the risk [36].

Some components of the innate and adaptive immune system may participate in the physiopathology of pre-eclampsia [37]. Although it is believed that placental hypoxia plays a relevant role in pre-eclampsia, recent studies suggest that the 
presence of hypoxic conditions may not be the key feature in all pre-eclamptic patients. The wall of the uterus is the territorial boundary between two genetically different individuals: the mother and the foetus. The uterine mucosal immune system appears to define this maternal/placental boundary. The decidua must control placentation, because in its absence, the trophoblast infiltrates to a dangerous extent, causing the condition of placenta accreta [38]. The decidua contains an abundant population of specialized natural killer (NK) cells. Placental NK cells, designated as uNK cells, play an important role in the acceptance and rejection of the foetus, as they are in direct contact with the trophoblasts [39]. uNK cells express killer-cell immunoglobulin-like receptors (KIRs) that recognize trophoblast HLA-C ligands [40]. Both KIR and HLA-C are genetically variable, resulting in many possible combinations of maternal KIR and foetal HLA-C ligands [41]. The KIR region is defined by two groups of haplotype: A and B. The KIR A haplotype has seven KIR genes, all encoding inhibitory receptors apart from KIR2DS4. In contrast, the KIR B haplotype contains a variable number of additional KIR, most of which encode activating receptors [42]. All HLA-C allotypes are KIR ligands and can be divided into two groups (carrying either $\mathrm{C} 1$ or $\mathrm{C} 2$ epitopes) that are distinguished by a dimorphism at position 80 and recognized by different KIRs [43]. A variety of diseases and clinical conditions has been associated with combinations of HLA-C and KIR genes. Case-control studies of pre-eclampsia performed both in pregnant European women [40, 44] and in sub-Saharan African women [45] showed that, when the foetus carries a $\mathrm{C} 2$ epitope, maternal KIR AA genotypes are risk factors for pre-eclampsia in both European and sub-Saharan African pregnant women, whereas different KIR B regions confer risk protection from pre-eclampsia in subSaharan African [45] and in European women [40, 44]. The KIR2DS1 gene of maternal KIR B haplotypes is protective in European women [40, 44]. The KIR2DS5 and KIR2DL1 genes confer risk and protection to pregnant SSA women [45].

\subsubsection{Implications for Birth Weight}

Functionally, the risky combination could result in very strong inhibition of uterine NK cells. Triggering of uterine NK cells by HLA-C2 target cells in vitro from women who have a protective KIR B haplotype (in which the activating KIR for HLA-C2, KIR2DS1, is located) results in secretion of cytokines and chemokines that may facilitate trophoblast invasion and vascular transformation limiting the highly invasive placenta while at the same time ensuring the foetus receives sufficient nourishment for normal development through remodelling of the spiral arteries [8]. A failure of the physiological transformation of uterine arteries is a common feature of all the great obstetric syndromes, and this results in a reduced placental supply of oxygen and nutrients, lower birth weights, and the risk of preterm labour and superimposed pre-eclampsia. However, at the same time, maternal and neonatal mortality is not only high under circumstances of reduced foetal nutrition but also when babies are too large for the pelvis. The optimal survival of babies weighing between 2.5 and $3.5 \mathrm{~kg}$ seems to be a universal feature of human populations. If 
babies become too large, the risk of prolonged obstructed labour, birth asphyxia, and postpartum haemorrhage is increased. Furthermore, these outcomes are much more common in African women with associated features of pregnancy that favour smaller babies: earlier birth (the gestational age is reduced to 38 weeks), the head engages late into the pelvis, and the baby matures earlier than in non-Africans [8]. The small neonatal size in ethnic minority groups is also, at least in part, due to persistent maternal constraints on foetal growth and can probably be regarded as mismatched when exposed to the current obesogenic environment. Thus, there is high mortality in mother and babies not only from pre-eclampsia (associated with low birth weight and still birth) but also at the other end of the normal birth weight spectrum. Both mothers and their babies benefit if the latter have intermediate birth weights and the two extremes of very low and high birth weight are selected against. The balance between these two extremes is partially determined at placentation, when uNK allows trophoblast cells to access sufficient maternal oxygen and nutrients without starving the baby (defective trophoblast invasion) or risking uterine rupture (excessive trophoblast invasion). In an African population, because of the greater risk of cephalopelvic disproportion, there is even greater selection for reduced foetal size with associated pre-eclampsia; this effect is consistent with the higher frequency of maternal KIR AA/paternal C2 combinations in SSA. In Europeans, opposing KIR/HLA-C combinations are associated with the extremes of birth weight: a paternal $\mathrm{C} 2$ epitope is associated with both extremes, but in preeclampsia and low birth weight (less than fifth centile), the risk is with maternal KIR AA genotypes, whereas in high birth weight, the association is with maternal KIR2DS1. Thus, there is a balance between the KIR A and KIR B haplotypes in both populations, but they differ in the regions of the KIR B haplotype that correlate with protection from pre-eclampsia. tB regions and KIR2DS1 are infrequent in Africans compared with Europeans, but the opposite is true for $\mathrm{cB}$ regions containing KIR2DS5. During the out-of-Africa migrations, it is possible that only individuals having tB with KIR2DS1 moved away from SSA. The higher Caesarean section rates seen in high-income countries in women of African ancestry may reflect not just delivery of women with pre-eclampsia but also an increased frequency of obstructed labour [46]. Furthermore, shoulder dystocia has also been reported to occur more commonly in African American women [47].

Thus, different KIR B regions protect sub-Saharan Africans and Europeans from pre-eclampsia, whereas in both populations, the KIR AA genotype is a risk factor for the syndrome. These results emphasize the importance of undertaking genetic studies of pregnancy disorders in African populations with the potential to provide biological insights not available from studies restricted to European populations.

\subsection{Gestational Diabetes Mellitus in Ethnic Minorities}

One consistent finding from studies from the Netherlands [48], Switzerland [49], and Norway [50] was that women originating from African and Middle Eastern countries tended to enter their pregnancies with higher BMI levels than the majority 
population in each country. All studies found, on the other hand, that pregnant women of South and East Asian descent overall were leaner than the European population. For instance, Asian/Asian British and Chinese/other had significantly higher OR for lean $(\mathrm{BMI}<18.5)$ vs. ideal weight compared with White British women [51]. However, these figures need to be interpreted considering studies showing substantial differences in the amount of body fat relative to BMI across ethnic groups, especially in Asians [52,53]. South Asians appear to be more insulin resistant than Europeans for the same level of BMI [54].

Pregnancy can be considered as a diabetogenic and inflammatory state due to the higher levels of maternal insulin resistance, hyperlipidaemia, and fat deposition. These normal alterations are aggravated by maternal adiposity. The insulin resistance in pregnancy increases about 50-60\% during pregnancy, irrespective of the prepregnant level. Thus, overweight and obese women start their pregnancy more insulin resistant compared with normal weight women and become highly insulin resistant in the second half of their pregnancy. This is also the case for pregnant women from East and South Asia, as they are found to be more insulin resistant compared with Western Europeans for the same level of BMI. Pancreatic B cells must compensate for the pregnancy-induced insulin resistance with increased insulin secretion. If not, hyperglycaemia may occur. South Asian women are reported to be less able to increase their B-cell function mutual to the pregnancy-induced insulin resistance compared with Western Europeans [55]. Reduced B-cell insulin response, in the setting of insulin resistance, is also seen among Asians outside pregnancy [56].

Gestational diabetes mellitus is defined as any degree of glucose intolerance with onset or first recognition during pregnancy [57, 58]. The different methodologies, diagnostic criteria, and screening practices make comparison of prevalence figures between populations a challenge $[59,60]$. In line with the trends for obesity and T2DM, increasing rates of GDM and undiagnosed T2DM in pregnant women attending antenatal care are observed in most parts of the world. A 2-3 higher OR for GDM was observed in women of South Asian origin [30, 50, 61-63]. The grouping of the ethnic minority population varies between studies, but also Black African [61], East Asian and Afro-Caribbean [30], and Middle Eastern [50] women had significantly higher OR for GDM.

The increasing trend for GDM globally, and the relatively higher susceptibility of many ethnic minority women in Europe today, is worrisome. GDM may reflect either a pre-existing, an undiagnosed T2DM, or a pregnancy-induced glucoseintolerant state with a high risk of future T2DM [64]. T2DM may be prevented or postponed in women after a pregnancy complicated by (GDM) and most clinical guidelines recommend a sustained screening program [65]. The time when the pregnancy GDM developed, obesity, insulin needs influence the gradual reduction in B-cell function and progression into T2DM [66]. Ethnicity is another parameter influencing the progression of the disease though the relation is complex and seems to differ between studies $[67,68]$. Recurrence rates of GDM in subsequent pregnancies vary between 30 and $84 \%$, with higher rates in minority groups compared with White populations and among those who do not develop GDM in a second pregnancy, had a reduced risk of T2DM [69]. 
A GDM pregnancy implies a substantially increased risk of future T2DM, although ethnic differences in conversion rates may exist. GDM may also imply a slight predisposition to later CVD. Regarding the neonates of women descending from Asian and African countries, the birth weights are generally lower than in the majority population. The small neonatal size in ethnic minority groups is most likely, at least in part, due to persistent maternal constraints on foetal growth and can probably be regarded as mismatched when exposed to the current obesogenic environment. Evidence is mounting that low birth weight increases the risk of later obesity, T2DM, and CVD. Furthermore, neonates exposed to maternal obesity and GDM may carry an increased risk of the same conditions linked to foetal overgrowth.

New public health initiatives need to focus more on early life interventions as interventions in adulthood have shown limited results. As breastfeeding may be associated with a decreased risk of obesity and adult disease both for the mother and baby, current efforts to promote breastfeeding should be strengthened, not least for ethnic minority groups.

\section{References}

1. World Health Organization. Trends in maternal mortality: 1990 to 2008. Geneva: World Health Organization; 2010.

2. Khan KS, Wojdyla D, Say L, et al. WHO analysis of causes of maternal death: a systematic review. Lancet. 2006;367:1066-74.

3. Alkema L, Chou D, Hogan D, et al. Global, regional, and national levels and trends in maternal mortality between 1990 and 2015, with scenario-based projections to 2030: a systematic analysis by the UN maternal mortality estimation inter-agency group. Lancet. 2016;387: 462-74.

4. Papaioannou GI, Syngelaki A, Maiz N, et al. Ultrasonographic prediction of early miscarriage. Hum Reprod. 2011;26:1685-92.

5. Salihu H, Kinniburgh BA, Aliyu MH, et al. Racial disparity in stillbirth among singleton, twin, and triplet gestations in the United States. Obstet Gynecol. 2004;104:734-40.

6. Sibai BM, Ewell M, Levine RJ, et al. Risk factors associated with preeclampsia in healthy nulliparous women. The calcium for preeclampsia prevention (CPEP) study group. Am J Obstet Gynecol. 1997;177:1003-10.

7. Caughey AB, Stotland NE, Washington AE, et al. Maternal ethnicity, paternal ethnicity, and parental ethnic discordance: predictors of preeclampsia. Obstet Gynecol. 2005;106:156-61.

8. Nakimuli A, Chazara O, Byamugisha J, et al. Pregnancy, parturition and preeclampsia in women of African ancestry. Am J Obstet Gynecol. 2014;210:510-520.e1.

9. Shen JJ, Tymkow C, MacMullen N. Disparities in maternal outcomes among four ethnic populations. Ethn Dis. 2005;15:492-7.

10. Yeung RO, Savu A, Johnson JA, et al. Ethnic differences in gestational diabetes mellitus-A population-level analysis. Diabetes. 2015;64:A19.

11. Yew TW, Khoo CM, Thai AC, et al. Diagnosis of gestational diabetes mellitus (GDM) using International Association of the Diabetes and Pregnancy Study Groups (IADPSG) cutoffs in a multi-ethnic Asian population. Diabetes. 2013;62:A359.

12. Carter MF, Fowler S, Holden A, et al. The late preterm birth rate and its association with comorbidities in a population-based study. Am J Perinatol. 2011;28:703-7.

13. Cohen GR, Curet LB, Levine RJ, et al. Ethnicity, nutrition, and birth outcomes in nulliparous women. Am J Obstet Gynecol. 2001;185:660-7. 
14. Frisbie WP, Biegler M, de Turk P, et al. Racial and ethnic differences in determinants of intrauterine growth retardation and other compromised birth outcomes. Am J Public Health. 1997;87:1977-83.

15. Poon LC, Karagiannis G, Stratieva V, et al. First-trimester prediction of macrosomia. Fetal Diagn Ther. 2011;29:139-47.

16. Gregory KD, Korst LM. Age and racial/ethnic differences in maternal, fetal, and placental conditions in laboring patients. Am J Obstet Gynecol. 2003;188:1602-6. discussion 1606-1608

17. Getahun D, Strickland D, Lawrence JM, et al. Racial and ethnic disparities in the trends in primary cesarean delivery based on indications. Am J Obstet Gynecol. 2009;201:422 e421-7.

18. Uzan J, Carbonnel M, Piconne O, et al. Pre-eclampsia: pathophysiology, diagnosis, and management. Vasc Health Risk Manag. 2011;7:467-74.

19. Khong TY, De Wolf F, Robertson WB, et al. Inadequate maternal vascular response to placentation in pregnancies complicated by pre-eclampsia and by small-for-gestational age infants. Br J Obstet Gynaecol. 1986;93:1049-59.

20. Redman CW, Sargent IL. Latest advances in understanding preeclampsia. Science. 2005;308:1592-4.

21. Ananth CV, Friedman AM. Ischemic placental disease and risks of perinatal mortality and morbidity and neurodevelopmental outcomes. Semin Perinatol. 2014;38:151-8.

22. Roberts JM. Pathophysiology of ischemic placental disease. Semin Perinatol. 2014;38:139-45.

23. Dekker GA. Risk factors for preeclampsia. Clin Obstet Gynecol. 1999;42:422-35.

24. Cabacungan ET, Ngui EM, McGinley EL. Racial/ethnic disparities in maternal morbidities: a statewide study of labor and delivery hospitalizations in Wisconsin. Matern Child Health J. 2012;16:1455-67.

25. Tanaka M, Jaamaa G, Kaiser M, et al. Racial disparity in hypertensive disorders of pregnancy in New York state: a 10-year longitudinal population-based study. Am J Public Health. 2007;97:163-70.

26. Brown HL, Chireau MV, Jallah Y, et al. The "Hispanic paradox": an investigation of racial disparity in pregnancy outcomes at a tertiary care medical center. Am J Obstet Gynecol. 2007;197:197.e1.

27. Knuist M, Bonsel GJ, Zondervan HA, et al. Risk factors for preeclampsia in nulliparous women in distinct ethnic groups: a prospective cohort study. Obstet Gynecol. 1998;92:174-8.

28. Zwart JJ, Jonkers MD, Richters A, et al. Ethnic disparity in severe acute maternal morbidity: a nationwide cohort study in the Netherlands. Eur J Pub Health. 2011;21:229-34.

29. Bouthoorn SH, Gaillard R, Steegers EA, et al. Ethnic differences in blood pressure and hypertensive complications during pregnancy: the generation R study. Hypertension. 2012;60: 198-205.

30. Khalil A, Rezende J, Akolekar R, et al. Maternal racial origin and adverse pregnancy outcome: a cohort study. Ultrasound Obstet Gynecol. 2013;41:278-85.

31. Poon LC, Kametas NA, Chelemen T, et al. Maternal risk factors for hypertensive disorders in pregnancy: a multivariate approach. J Hum Hypertens. 2010;24:104-10.

32. Klungsoyr K, Morken NH, Irgens L, et al. Secular trends in the epidemiology of pre-eclampsia throughout 40 years in Norway: prevalence, risk factors and perinatal survival. Paediatr Perinat Epidemiol. 2012;26:190-8.

33. Mogren I, Hogberg U, Winkvist A, et al. Familial occurrence of preeclampsia. Epidemiology. 1999;10:518-22.

34. Skjaerven R, Vatten LJ, Wilcox AJ, et al. Recurrence of pre-eclampsia across generations: exploring fetal and maternal genetic components in a population based cohort. BMJ. 2005;331:877.

35. Shahabi A, Wilson ML, Lewinger JP, et al. Genetic admixture and risk of hypertensive disorders of pregnancy among Latinas in Los Angeles County. Epidemiology. 2013;24:285-94.

36. Esplin MS, Fausett MB, Fraser A, et al. Paternal and maternal components of the predisposition to preeclampsia. N Engl J Med. 2001;344:867-72.

37. Laresgoiti-Servitje E. A leading role for the immune system in the pathophysiology of preeclampsia. J Leukoc Biol. 2013;94:247-57. 
38. Hannon T, Innes BA, Lash GE, et al. Effects of local decidua on trophoblast invasion and spiral artery remodeling in focal placenta creta - an immunohistochemical study. Placenta. 2012;33:998-1004.

39. Guleria I, Sayegh MH. Maternal acceptance of the fetus: true human tolerance. J Immunol. 2007;178:3345-51.

40. Hiby SE, Apps R, Sharkey AM, et al. Maternal activating KIRs protect against human reproductive failure mediated by fetal HLA-C2. J Clin Invest. 2010;120:4102-10.

41. Parham P, Moffett A. Variable NK cell receptors and their MHC class I ligands in immunity, reproduction and human evolution. Nat Rev Immunol. 2013;13:133-44.

42. Parham P. Immunogenetics of killer cell immunoglobulin-like receptors. Mol Immunol. 2005;42:459-62.

43. Mandelboim O, Reyburn HT, ValesGomez M, et al. Protection from lysis by natural killer cells of group 1 and 2 specificity is mediated by residue 80 in human histocompatibility leukocyte antigen $\mathrm{C}$ alleles and also occurs with empty major histocompatibility complex molecules. $\mathrm{J}$ Exp Med. 1996;184:913-22.

44. Hiby SE, Walker JJ, O'Shaughnessy KM, et al. Combinations of maternal KIR and fetal HLA-C genes influence the risk of preeclampsia and reproductive success. J Exp Med. 2004;200:957-65.

45. Nakimuli A, Chazara O. A KIR B centromeric region present in Africans but not Europeans protects pregnant women from pre-eclampsia, vol. 112; 2015. p. 845-50.

46. Malin M, Gissler M. Maternal care and birth outcomes among ethnic minority women in Finland. BMC Public Health. 2009;9:84.

47. Cheng YW, Norwitz ER, Caughey AB. The relationship of fetal position and ethnicity with shoulder dystocia and birth injury. Am J Obstet Gynecol. 2006;195:856-62.

48. Djelantik AA, Kunst AE, van der Wal MF, et al. Contribution of overweight and obesity to the occurrence of adverse pregnancy outcomes in a multi-ethnic cohort: population attributive fractions for Amsterdam. BJOG. 2012;119:283-90.

49. Loetscher KC, Selvin S, Zimmermann R, et al. Ethnic-cultural background, maternal body size and pregnancy outcomes in a diverse Swiss cohort. Women Health. 2007;45:25-40.

50. Jenum AK, Morkrid K, Sletner L, et al. Impact of ethnicity on gestational diabetes identified with the WHO and the modified International Association of Diabetes and Pregnancy Study Groups criteria: a population-based cohort study. Eur J Endocrinol. 2012;166:317-24.

51. Heslehurst N, Rankin J, Wilkinson JR, et al. A nationally representative study of maternal obesity in England, UK: trends in incidence and demographic inequalities in 619323 births, 1989-2007. Int J Obes. 2010;34:420-8.

52. Deurenberg P, Deurenberg-Yap M, Guricci S. Asians are different from Caucasians and from each other in their body mass index/body fat per cent relationship. Obes Rev. 2002;3:141-6.

53. Barba C, Cavalli-Sforza T, Cutter J, et al. Appropriate body-mass index for Asian populations and its implications for policy and intervention strategies. Lancet. 2004;363:157-63.

54. Misra A, Khurana L. Obesity-related non-communicable diseases: south Asians vs white Caucasians. Int J Obes. 2011;35:167-87.

55. Morkrid K, Jenum AK, Sletner L, et al. Failure to increase insulin secretory capacity during pregnancy-induced insulin resistance is associated with ethnicity and gestational diabetes. Eur J Endocrinol. 2012;167:579-88.

56. Golden SH, Brown A, Cauley JA, et al. Health disparities in endocrine disorders: biological, clinical, and nonclinical factors - an Endocrine Society scientific statement. J Clin Endocrinol Metab. 2012;97:E1579-639.

57. International Association of Diabetes and Pregnancy Study Groups Consensus Panel, Metzger $\mathrm{BE}$, et al. International association of diabetes and pregnancy study groups recommendations on the diagnosis and classification of hyperglycemia in pregnancy. Diabetes Care. 2010;33:676-82.

58. Alberti KG, Zimmet PZ. Definition, diagnosis and classification of diabetes mellitus and its complications. Part 1: diagnosis and classification of diabetes mellitus provisional report of a WHO consultation. Diabet Med. 1998;15:539-53. 
59. Buckley BS, Harreiter J, Damm P, et al. Gestational diabetes mellitus in Europe: prevalence, current screening practice and barriers to screening. A review. Diabet Med. 2012;29:844-54.

60. Sexton H, Heal C, Banks J, et al. Impact of new diagnostic criteria for gestational diabetes. J Obstet Gynaecol Res. 2018;44:425.

61. Makgoba M, Savvidou MD, Steer PJ. An analysis of the interrelationship between maternal age, body mass index and racial origin in the development of gestational diabetes mellitus. BJOG. 2012;119:276-82.

62. Agarwal MM, Dhatt GS, Shah SM. Gestational diabetes mellitus simplifying the International Association of Diabetes and Pregnancy diagnostic algorithm using fasting plasma glucose. Diabetes Care. 2010;33:2018-20.

63. Jiwani A, Marseille E, Lohse N, et al. Gestational diabetes mellitus: results from a survey of country prevalence and practices. J Matern Fetal Neonatal Med. 2012;25:600-10.

64. Kim C, Berger DK, Chamany S. Recurrence of gestational diabetes mellitus: a systematic review. Diabetes Care. 2007;30:1314-9.

65. Ratner RE, Christophi CA, Metzger BE, Dabelea D, Bennett PH, Pi-Sunyer X, et al. Prevention of diabetes in women with a history of gestational diabetes: effects of metformin and lifestyle interventions. J Clin Endocrinol Metabol. 2008;93(12):4774-9.

66. Tovar A, Chasan-Taber L, Eggleston E, Oken E. Postpartum screening for diabetes among women with a history of gestational diabetes mellitus. Prev Chronic Dis. 2011;8:A124.

67. Kim C, Newton KM, Knopp RH. Gestational diabetes and the incidence of type 2 diabetes: a systematic review. Diabetes Care. 2002;25(10):1862-8.

68. Retnakaran R, Austin PC, Shah BR. Effect of subsequent pregnancies on the risk of developing diabetes following a first pregnancy complicated by gestational diabetes: a population based study. Diabet Med. 2011;28:28792.

69. Jenum AK, Sommer C, Sletner L, Mørkrid K, Bærug A, Mosdøl A. Adiposity and hyperglycaemia in pregnancy and related health outcomes in European ethnic minorities of Asian and African origin: a review. Food Nutr Res. 2013;57 